\title{
Emerging role of PR domain containing 5 (PRDM5) as a broad tumor suppressor in human cancers
}

\author{
Xing-sheng Shu
}

Received: 23 August 2014 / Accepted: 28 November 2014 / Published online: 14 December 2014

(C) International Society of Oncology and BioMarkers (ISOBM) 2014

To the editor,

An excellent article titled "Promoter methylation-mediated downregulation of PRDM5 contributes to the development of lung squamous cell carcinoma" was published recently in Tumor Biology. This study illustrated the epigenetic silencing of PRDM5 tumor suppressor in lung cancer cells and revealed the potential diagnostic and prognostic value of PRDM5 methylation for lung cancer patients [1]. This work, together with ours and others', highlights the emerging role of PRDM5 as a broad tumor suppressor disrupted by promoter hypermethylation in common cancers (Table 1).

A decade ago, Huang and colleagues isolated the fulllength cDNA of PRDM5 gene, and they showed for the first time that the $\mathrm{CpG}$ island within PRDM5 promoter region was frequently methylated in breast, ovarian, and liver cancers [2]. Since then, epigenetic inactivation of PRDM5 has been documented in a broad spectrum of solid tumor types, which originate from various organs of the human body, including digestive system tumors (esophageal, gastric, colorectal), respiratory system tumors (nasopharyngeal, lung), gynecologic tumors (ovarian, cervical), as well as liver and breast tumors. These findings strongly implicate that loss of PRDM5 is a common molecular event during tumorigenesis, and PRDM5 could be a critical tumor suppressor.

Through independent studies, we and other groups confirmed the causal relationship between PRDM5 promoter hypermethylation and its downregulation in cancer cells [1-5]. Intriguingly, in addition to DNA methylation, another epigenetic mechanism is also involved in PRDM5 silencing.

X.-s. Shu $(\bowtie)$

Institute of Molecular Medicine, Health Science Center, Shenzhen University, Shenzhen 518060, China

e-mail: shu@szu.edu.cn
Watanabe et al. found that in colon cancer cells with sparse PRDM5 methylation, a repressive histone modification, trimethylation of $\mathrm{H} 3 \mathrm{~K} 27$, was enriched in its promoter region, contributing to its transcriptional silencing [4]. In fact, recent advances in epigenetics field underscore the interplay between DNA methylation and histone modification, in which histone modifications create a "flexible" state around transcription start site while DNA methylation can "lock" the state of transcription permanently [6]. PRDM5 here gives us an excellent example of this cooperating epigenetic regulation. Apart from epigenetic alterations, classic tumor suppressor genes usually bear genetic abnormalities in cancer. Although no genetic mutation of PRDM5 in tumor is reported thus far, searching the COSMIC database (catalog of somatic mutation in cancer) did reveal low-frequency point mutations within PRDM5 gene coding region in most types of cancer, suggesting PRDM5 mutation may as well contribute to tumor formation. However, the functional relevance of these mutations remains to be delineated.

To understand how PRDM5 exerts its tumor-suppressive function, Deng and Huang first demonstrated that overexpression of PRDM5 induced cell cycle G2/M arrest and apoptosis of cancer cells [2]. Further studies showed that inhibition of certain oncogenic pathways, such as Wnt signaling, by PRDM5 might be a crucial part of its tumor-suppressive activity [3, 7]. As an epigenetic master gene, PRDM5 does not possess intrinsic histone methyltransferase (HMT) activity, but it can recruit another HMT G9a to the promoter region of target genes and thereby regulates their transcription [8]. In addition, a recent proteomic study revealed PRDM5 could also interact with insulator factors such as CTCF to modulate chromatin organization of target genome sites [9]. Very recently, a wonderful mouse model work by Lund and colleagues showed knockout of $\operatorname{Prdm} 5$-accelerated $A p c^{\mathrm{Min}}$-driven intestinal carcinogenesis [10]. Furthermore, they 
Table $1 \quad P R D M 5$ methylation in various types of cancer

\begin{tabular}{|c|c|c|c|c|}
\hline \multirow[t]{2}{*}{ Type of cancer } & \multicolumn{3}{|c|}{ Methylation percentage } & \multirow[t]{2}{*}{ Reference(s } \\
\hline & Cell lines & Tumor tissues & Normal tissues & \\
\hline Esophageal & $44.4 \%(8 / 18)$ & $58.1 \%(25 / 43)$ & $42.8 \%(3 / 7)$ & {$[3]$} \\
\hline Gastric (2007) & $80.0 \%(4 / 5)$ & $50.0 \%(39 / 78)$ & $0 / 15$ & {$[4]$} \\
\hline Gastric (2011) & $76.5 \%(13 / 17)$ & $88.1 \%(37 / 42)$ & N/A & {$[3]$} \\
\hline Colorectal & $50.0 \%(1 / 2)$ & $6.6 \%(4 / 61)$ & N/A & {$[4]$} \\
\hline Liver (2004) & $87.5 \%(7 / 8)$ & $52.8 \%(19 / 36)$ & $0 / 3$ & {$[2]$} \\
\hline Liver (2011) & $25.0 \%(3 / 12)$ & $63.0 \%(29 / 46)$ & $0 / 9$ & {$[3]$} \\
\hline Nasopharyngeal & $80.0 \%(4 / 5)$ & $93.5 \%(43 / 46)$ & $28.6 \%(2 / 7)$ & [3] \\
\hline Lung & $28.6 \%(2 / 7)$ & $73.3 \%(22 / 30)$ & $46.7 \%(14 / 30)$ & {$[1,3]$} \\
\hline Breast & $80.0 \%(8 / 10)$ & $52.5 \%(21 / 40)$ & $0 / 3$ & {$[2]$} \\
\hline Ovarian & $83.3 \%(5 / 6)$ & N/A & N/A & {$[2]$} \\
\hline Cervical & $75.0 \%(3 / 4)$ & $40.5 \%(17 / 42)$ & $0 / 9$ & {$[5]$} \\
\hline
\end{tabular}

$N / A$ not tested

systematically profiled the genome-wide occupancy of PRDM5 and identified a subset of metabolic genes as its direct targets, which may function as downstream effectors of PRDM5-mediated tumor suppression.

Epigenetic silencing of tumor suppressor genes, especially by promoter hypermethylation, is believed to occur at early stages of tumor development [11], and the power of DNA methylation as biomarker for early detection has been extensively explored [12]. As summarized in Table 1, PRDM5 methylation is frequently detected in primary tumor tissues (except colorectal cancer). In contrast, the percentages of PRDM5 methylation in normal tissues are much lower, suggesting it is a potential epigenetic biomarker for these tumors. However, although the detection sensitivity of PRDM5 methylation is high (above $50 \%$ for most tumors), its specificity in certain types of cancer (esophageal and lung cancers) is poor, and the number of normal tissues tested in these studies is very limited. In addition, methylation-specific PCR (MSP) data of PRDM5 methylation in the same cancer type (e.g., liver cancer) varies in different studies, probably due to use of different MSP primers (methylation status of individual $\mathrm{CpG}$ sites can be different from each other in the same sample) and variable amplification power of different MSP reactions. Thus, including large-scale paired normal/cancer tissues and premalignant samples (if possible) and adopting more accurate and sensitive detection method (e.g., quantitative MSP) in future studies will be very helpful to comprehensively evaluate the value of PRDM5 methylation as a biomarker.

Acknowledgments This work was supported by the National Natural Science Foundation of China (81301783) and Natural Science Foundation of Shenzhen University (201409).

\section{Conflicts of interest None.}

\section{References}

1. Tan SX, Hu RC, Tan YL, Liu JJ, Liu WE. Promoter methylationmediated downregulation of PRDM5 contributes to the development of lung squamous cell carcinoma. Tumour Biol: J Int Soc Oncodev Biol Med. 2014;35(5):4509-16. doi:10.1007/ s13277-013-1593-2.

2. Deng Q, Huang S. PRDM5 is silenced in human cancers and has growth suppressive activities. Oncogene. 2004;23(28):4903-10. doi: 10.1038/sj.onc.1207615.

3. Shu XS, Geng H, Li L, Ying J, Ma C, Wang Y, et al. The epigenetic modifier PRDM5 functions as a tumor suppressor through modulating WNT/beta-catenin signaling and is frequently silenced in multiple tumors. PLoS One. 2011;6(11):e27346. doi:10.1371/journal. pone.0027346.

4. Watanabe Y, Toyota M, Kondo Y, Suzuki H, Imai T, Ohe-Toyota M, et al. PRDM5 identified as a target of epigenetic silencing in colorectal and gastric cancer. Clin Cancer Res: Off J Am Assoc Cancer Res. 2007;13(16):4786-94. doi:10.1158/10780432.CCR-07-0305.

5. Cheng HY, Chen XW, Cheng L, Liu YD, Lou G. DNA methylation and carcinogenesis of PRDM5 in cervical cancer. J Cancer Res Clin Oncol. 2010;136(12):1821-5. doi:10.1007/ s00432-010-0840-9.

6. Easwaran H, Tsai HC, Baylin SB. Cancer epigenetics: tumor heterogeneity, plasticity of stem-like states, and drug resistance. Mol Cell. 2014;54(5):716-27. doi:10.1016/j.molcel. 2014.05.015.

7. Meani N, Pezzimenti F, Deflorian G, Mione M, Alcalay M. The tumor suppressor PRDM5 regulates Wnt signaling at early stages of zebrafish development. PLoS One. 2009;4(1):e4273. doi:10.1371/ journal.pone.0004273.

8. Duan Z, Person RE, Lee HH, Huang S, Donadieu J, Badolato R, et al. Epigenetic regulation of protein-coding and microRNA genes by the Gfil-interacting tumor suppressor PRDM5. Mol Cell Biol. 2007;27(19):6889-902. doi:10.1128/MCB. 00762-07.

9. Galli GG, Carrara M, Francavilla C, de Lichtenberg KH, Olsen JV, Calogero RA, et al. Genomic and proteomic analyses of Prdm5 reveal interactions with insulator binding proteins in embryonic stem cells. Mol Cell Biol. 2013;33(22):4504-16. doi:10.1128/MCB. 00545-13. 
10. Galli GG, Multhaupt HA, Carrara M, de Lichtenberg KH, Christensen IB, Linnemann D, et al. Prdm5 suppresses Apc(Min)driven intestinal adenomas and regulates monoacylglycerol lipase expression. Oncogene. 2014;33(25):3342-50. doi:10.1038/onc. 2013.283
11. Baylin SB, Ohm JE. Epigenetic gene silencing in cancer-a mechanism for early oncogenic pathway addiction? Nat Rev Cancer. 2006;6(2):107-16. doi:10.1038/nrc1799.

12. Laird PW. The power and the promise of DNA methylation markers. Nat Rev Cancer. 2003;3(4):253-66. doi:10.1038/nrc1045. 